\title{
Clinical Analysis of Posterolateral Approach for the Treatment of Posterior Malleolus Fracture with Cannulated Screw or Buttress Plate
}

Yang Lei

Jiangsu University Affiliated Wujin Hospital: Changzhou Wujin People's Hospital

liu zhiyuan ( icenatureback@163.com )

Changzhou Wujin People's Hospital

\section{Yin Gang}

Jiangsu University Affiliated Wujin Hospital: Changzhou Wujin People's Hospital

\section{Research}

Keywords: Posterior malleolus fracture, Posterolateral approach, Lag screws, Buttress plates

Posted Date: June 18th, 2021

DOl: https://doi.org/10.21203/rs.3.rs-609807/v1

License: (1) This work is licensed under a Creative Commons Attribution 4.0 International License.

Read Full License 


\section{Abstract}

\section{Background:}

Posterior malleolus (PM) fractures account for $7 \%-44 \%$ of all ankle fractures. however, the management of PM fractures remains controversial. Studies have shown that the posterolateral approach is one of the most commonly used surgical approaches. The aim of this study was to evaluate the clinical effect of the posterolateral approach with cannulated screw or buttress plate for the treatment of posterior malleolus fracture.

\section{Method:}

We retrospectively analyzed the clinical data of 66 patients with ankle fractures involving posterior malleolus from January 2016 to March 2018. All patients were treated with a posterolateral approach. Fixation of the posterior malleolus was made with anterior to posterior (AP) lag screws in 7 patients, posterior to anterior (PA) lag screws in 38 patients, buttress plates in 9 patients, and buttress plates combined with PA lag screws in 12 patients. We used the AOFAS ankle and posterior foot function scoring system, VAS pain score, and radiographic evaluations as the primary outcome measures. The mean follow-up was $10.8 \pm 4.4$ (range, 6-20) months.

\section{Results:}

Radiological evaluation showed that 64 patients (97.0\%) achieved a good or excellent reduction and the primary bone union was achieved in all the 66 patients without internal fixation failure or occurrence of post-traumatic ankle arthritis. At the final follow-up, the mean AOFAS score of the patients was $92.39 \pm$ 3.84 , with an excellent/good rate of $100 \%$. The VAS pain score was $6.62 \pm 1.03$ before surgery, changed to $3.06 \pm 0.72$ one week after surgery, and $1.20 \pm 0.92$ at the final follow-up. There was no statistical difference in the AOFAS score $(p=0.01)$ or VAS pain score $(p=0.01)$ between the different internal fixation methods.

\section{Conclusion:}

The posterolateral approach using lag screws and/or buttress plates can achieve good clinical outcomes in the treatment of posterior malleolus fracture with reduced incidence of postoperative complications, fracture reduction failure, and ankle osteoarthritis.

\section{Introduction}

Ankle fractures are a relatively common type of limb fracture, while posterior malleolus (PM) fractures account for $7 \%-44 \%$ of all ankle fractures[1]. Although there is specific treatment recommended for medial and lateral malleolus fractures, the management of PM fractures remains controversial. 
After reduction of the lateral malleolus, the PM fragment might achieve spontaneous reduction with the auxiliary effect of the posterior tibiofibular ligament. It is widely accepted that the fixation of the PM fracture requires further open treatment when the area of the posterior marginal fragment is more than $25 \%$ of the articular surface or when the ankle joint remains unstable after medial and lateral malleolus fixation [2, 3]. Drijfhou and Verhage found that medium-sized fragments of the posterior malleolus (5$25 \%$ ) may increase the incidence of posttraumatic radiographic osteoarthritis. Furthermore, radiographic osteoarthritis also occurred more frequently when postoperative step-off was $1 \mathrm{~mm}$ or more[4]. Studies have shown that anatomical reduction and internal fixation of PM fractures is an important functional outcome in the treatment of ankle fractures $[5,6]$.

The choice of treatment depends on the shape, size, displacement of the fragments, and classification of ankle fractures. Generally, PM fragments are fixed either with indirect percutaneous screws or through a posterolateral approach using screws and/or a buttress plate.

In this study, we assessed the short-term clinical outcome in 66 patients with PM ankle fracture using a posterolateral approach. We also compared the outcomes of different fixation methods (screw and/or buttress plates).

\section{Patients And Methods}

This retrospective comparative study was conducted at a level one trauma center.

\section{Patients}

Patients with ankle fracture were screened to identify candidates meeting inclusion criteria of (1) ankle fracture involving the PM, (2) aged 18 years and above, (3) PM was surgically fixed with lag screws and/or buttress plates via a posterolateral approach. Patients were excluded if they had tibia pilon fractures, open fractures, history of ankle fractures, and pathological fractures. A total of 66 patients met the inclusion criteria and were recruited between January 2016 and March 2018. Approval for the study was obtained from our Institutional Review Board. Patients were classified according to the LaugeHansen and Haraguchi classification system with the help of preoperative AP, lateral x-rays, and 3D CT scans. All fractures were unilateral, with a mean follow-up of 10.8 \pm 4.4 (range, 6-20) months.

Baseline data collection included demographic characteristics, Lauge-Hansen and Haraguchi classification of the fracture, and injury mechanism as shown in Table 1.

\section{Operative Procedure}

The patients were treated with lag screws and/or buttress plates. Patients were either in the prone or lateral position. A posterolateral approach was performed through the interval between the peroneal tendons and flexor hallucis longus to gain access to the posterior malleolus. Care is taken to avoid injury to the sural nerve, flexor hallucis longus, and the posterior inferior tibiofibular ligament. When anatomical 
reduction was confirmed with image intensification, the PM was reduced directly and held temporarily with $\mathrm{K}$ wires or a pointed reduction clamp.

In the anteroposterior (AP) or posteroanterior (PA) lag screw group, 1 or 2 percutaneous $3.5-\mathrm{mm}$ lag screws were placed from anterior to posterior (A to $P$ ) or anterior to posterior ( $P$ to $A$ ) into the PM (Fig. 1 and Fig. 2). In the buttress plates group, PM was fixed with either a small fragment $T$ plate or $1 / 3$ tubular plate applied in a buttress technique. In the buttress plates and lag screws group, 1 or 2 lag screws were placed under the plates and just close to the articular surface except for the plates (Fig. 3).

Medial malleolus fracture was treated after PM in supine position with lag screws.

The tibiofibular syndesmosis (TFS) was fixed with a syndesmosis screw or endobutton system if tibiofibular instability after fixation of the lateral, medial, and PM fractures was present.

The postoperative treatment of ankle fractures was to allow a range of motion (ROM) and stretching exercise after surgery. Non-weight-bearing continued for at least six weeks until there was radiographic evidence of fracture healing. All patients were instructed to begin weight-bearing at 6 weeks aiming for full-weight bearing by 12 weeks. The TFS screw was removed before full weight bearing to prevent screw breakage.

\section{Surgical Outcome Evaluation}

Patients were evaluated by clinical examination and radiography.

First, we identified any surgical complications after the operation. Articular step-off and/or articular surface gap was assessed using postoperative plain radiographs or CT scan. The reduction was considered excellent $(<1 \mathrm{~mm})$, good $(1-2 \mathrm{~mm})$, and poor $(>2 \mathrm{~mm})$ as proposed by Ketz[7]. Radiographic images were analyzed for the loss of reduction, bone union rate, and arthritic changes of the ankle joint at the last follow-up. The Visual Analog Scale (VAS) was used to quantify pain before surgery, one week after surgery, and before removing the internal fixation. The functional outcomes of all patients were evaluated using the American Orthopaedic Foot and Ankle Society (AOFAS) scores at the final follow-up.

\section{Statistical Analysis}

SPSS 20.0 was used for statistical analysis.

Kolmogorov-Smirnov test was used to assess whether the VAS pain scores in all 66 patients at different times were normally distributed and the scores were further compared using the Rank sum test. A oneway ANOVA test was used to compare the AOFAS scores of different internal fixation methods. VAS pain scores at different time points were analyzed by Rank sum test with Bonferroni correction. Besides, a Chisquared test was used to determine whether there was an association between categorical variables, including sex, left/right side, mechanism of injury, Lauge-Hansen classification, Haraguchi classification, 
and the AOFAS or VAS scores for each group. Correlations of categorical variables including age, followup period were also assessed using one-way ANOVA test or Rank sum test, respectively.

A p-value $<0.05$ was considered statistically significant.

\section{Results}

The AP or PA lag screws were used to fix the fragment was fixed using in 45 patients (68.2\%), while in 21 patients (31.8\%), it was fixed using a buttress plate with or without lag screws. After fixation, 45 patients $(68.2 \%)$ were found to have stable ankles. A syndesmosis screw (17 cases) or endobutton system (4 cases) was required in $31.8 \%$ of the patients. No intraoperative injuries of the sural nerve, peroneal artery, and tendons were reported. Postoperative complications such as wound infection, soft tissue necrosis, adhesion of flexor hallucis longus were not reported in any of the patients.

Radiographic evaluation revealed excellent reduction with a congruent ankle joint in 59 cases (89.4\%), 5 cases had good reduction (7.6\%), while $2(3.0 \%)$ cases had poor reduction (Table $2-1)$. No invalidation or breakage of internal fixation had occurred after an average follow-up period of $10.8 \pm 4.4$ months, and all patients achieved primary bone union. Besides, no traumatic osteoarthritis was reported in any of the participating patients.

The mean AOFAS score for the evaluation of ankle function at the last follow-up was $92.39 \pm 3.84$. Besides, 52 patients have an excellent score (90-100 points), 14 patients got a good score (75-89 points), and none of the patients had an AOFAS score below 75 (Table $2-1$ ).

The mean VAS scores of the patients in the buttress plate group were $6.62 \pm 1.03,3.06 \pm 0.72$, and $1.20 \pm$ 0.92 before the operation, one week after surgery, and before removing the internal fixation, respectively (Table 2-2). Patients had significantly lower VAS scores one week after surgery $(P<0.05)$ or at the last follow-up $(P<0.05)$.

We further explored the clinical outcome of the different internal fixation methods. The 66 Patients were divided into three groups as follows; AP screw group, PA screw group, and buttress plates group. The demographic characteristics, mechanism of injury, classification of the fracture, and follow-up times of the patient cohorts were similar (Table 3, P > 0.05). Radiological evaluation of the ankle showed that excellent or good reduction was achieved in $85.7 \%, 97.4 \%$, and $100 \%$ of patients in the AP screw group, PA screw group, and buttress plates group, respectively. Besides, 1, 13, and 7 patients in the AP screw group, PA screw group, and buttress plates group, respectively, needed additional syndesmosis screw or endobutton system as shown in Table $4-1$. The AOFAS and VAS scores among different groups are listed in Table 4 - 2. No statistically significant difference in mean AOFAS scores at the final follow-up was noted or VAS scores before operation and before removing the internal fixation for each group $(P>$ 0.05). However, for the VAS scores after the operation, the PA screw group reported slightly higher scores than the AP screw group $(P<0.05)$ 


\section{Discussion}

Posterior malleolus fractures refer to ankle fractures of the posterior articular surface of the distal tibia. A previous study reported that fractures of the posterior margin of the distal tibia occurred in $46 \%$ of DanisWeber Type B and Type C [8]. The posterior ankle fracture is also believed to participate in the symmetrical coordination of the joint and flatness of the articular surface [9]. Compared with medial and lateral malleolus fractures, ankle fractures with posterior malleolus or Volkmann bone fragments often have ankle instability and subluxation, which can lead to a worse prognosis.

Until recently, the treatment of posterior malleolus fracture has remained controversial [10]. A systematic review was conducted on the biomechanical and clinical evaluation of posterior malleolar fractures. The study included eight biomechanical studies and 10 clinical studies, involving 96 cadaveric ankles and 447 fractured ankles. However, this study found no consensus in the literature on which fragment size of the posterior malleolus should be internally fixed [11]. For a long time, posterior malleolar fracture fixation was often performed only when the fragment size exceeded $1 / 4$ to $1 / 3$ of the joint surface based on biomechanical, cadaveric, and small population clinical studies[3, 12, 13]. However, the fixation of posterior malleolar fractures provided greater syndesmotic stability and was beneficial for the reduction of distal fibula fracture [6]. Recent studies have also demonstrated that radiographic osteoarthritis is increased in patients with medium (5-25\%) and large (>25\%) posterior fragments than in those with small fragments.

Special types and irregular fracture lines affected the judgment of fragment size and displacement. The CT scan allowed us to infer the specific shape and size of the fracture fragments and whether the fracture accumulated on the fibula notch or medial malleolus at the same time. Haraguchi classification is important for accurate diagnosis of posterior malleolus fractures and determination of appropriate surgical approaches [14]. It is generally believed that type I and type II are often associated with posterior medial malleolus fractures and larger fragments. ORIF is often required to reconstruct the articular surface and restore the stability of the ankle joint. In the present study, we evaluated the severity of posterior medial malleolus fractures based on Lauge-Hansen and Haraguchi classification. Based on the Lauge-Hansen classification system, the most common type of ankle fracture was the supinationexternal rotation pattern $(46 ; 70 \%)$, followed by pronation external rotation $(16 ; 24 \%)$, while based on the Haraguchi classification system, 38 fractures were type I fractures (58\%) and 24 were type II fractures (36\%).

Internal fixation of posterior malleolus fractures may be performed either through indirect or direct techniques. At present, the most commonly used surgical techniques are indirect reduction by anterior-toposterior fixation, direct reduction, and fixation from the posterolateral or posteromedia approach. For each specific patient, the choice of the approach depends on the type of posterior malleolus fracture and the overall malleolar fracture pattern. The experience of the surgeon is also an important factor in surgical decision-making [15]. Indirect reduction and anteroposterior (AP) fixation have better traumatic control and fewer complications. On the other hand, direct reduction via open surgery and posteroanterior 
(PA) fixation have more accurate reduction and superior biomechanical strength. In our study, direct reduction via a posterolateral approach achieved a $97 \%$ excellent and good reduction rate. Timothy $\mathrm{J}$, et al performed a retrospective study of radiographic and clinical midterm outcomes of posterior malleolar fractures treated with AP lag screws or posterior buttress plating. The plating group demonstrated superior postoperative SMFA scores compared with the AP screw group. The study also reported significant differences in the SMFA bother index and trends toward improvement in the mobility and functional indices [16]. A recent study evaluated the outcomes of indirect reduction with AP fixation versus direct reduction with PA fixation via a posterolateral approach in 46 patients with posterior malleolus fixation. The findings revealed that the direct reduction technique and PA fixation enhanced the quality of reduction and led to better functional outcomes in the management of the posterior fragment involving more than $25 \%$ of the articular surface, displacement by more than $2 \mathrm{~mm}$, and ankle instability [17].

The posterolateral approach is one of the most commonly used surgical approaches for the treatment of posterior malleolus fractures [18-20]. With this approach fractures of the posterior malleolus and the lateral malleolus can be reduced and fixed via the same incision. It is especially useful with relatively small posterior malleolus fragments and in the presence of additional fragments that cannot be indirectly reduced [21, 22]. In this study, we confirmed that the posterolateral approach has the advantages of adequate fracture exposure, accurate reduction, and precise fixation. In this study, $97 \%$ of the patients reported a good or excellent reduction. Besides, this approach had a relatively low postoperative complication rate. The ORIF with buttress plates and lag screws via a posterolateral approach was found to be an effective technique based on the AOFAS and VAS scores, with satisfactory functional recovery and unperceivable pain after surgery.

Another area of debate regarding fixation of the posterior malleolus fracture is the internal fixation materials or inner plants to use with the same posterolateral approach. Mehmet, et al prospectively evaluated the clinical results of 40 patients with posterior malleolar fracture between 2008 and 2012. The patients were treated with a posterolateral approach and received either plate or screw fixation. The results showed no statistically significant difference in AOFAS scores and ROM of the ankle between the two groups [23]. Compared with screw fixation, a buttress plate can provide a mechanical environment for fracture stability, resist axial stress, and shear force, which is important for ankle joint congruity and stability. A biomechanical study in a human cadaveric model confirmed that the buttress plate group showed less peak axial displacement during cyclic loading test and less permanent axial displacement beginning at cycle 200, although there were no significant differences between the AP lag screws group and buttress plate group during load-to-failure testing [24]. Supination external rotation (SER) injury is always accompanied by posterior malleolar fracture and syndesmosis instability. Mengnai et al investigated the correlation between posterior malleolar fracture fixation and the need for additional syndesmotic screw fixation. When the posterior malleolar fragment in SER IV ankle fractures was fixated with a direct posterior lateral plate, the need for syndesmotic fixation was reduced [25]. Specifically, for large posterior malleolus fractures, a posterolateral approach with buttress plate and cannulated screw internal fixation can achieve anatomic reduction and primary bone union without displacement, 
loosening, or breakage of internal fixation. Besides, according to the Baird-Jackson scoring system, approximately $82.3 \%$ of patients reported a high degree of satisfaction after surgery [26].

In this study, using a posterolateral approach, we fixed posterior malleolus fracture using AP lag screws, PA lag screws, buttress plates only, or with PA lag screws. First, the excellent or good reduction rates were $85.7 \%, 97.4 \%$, and $100 \%$, respectively, in the three groups. A total of 21 cases had unstable distal tibiofibular syndesmosis after posterior malleolar fixation, while $14.3 \%, 34.2 \%$, and $33.3 \%$ of patients in the AP lag screws, PA lag screws, buttress plates only, or with PA lag screws groups needed additional syndesmotic fixation with lag screws or endobutton system. There was no significant difference in AOFAS scores among the different groups. However, for VAS scores, the PA group had a slightly higher score compared with the AP group after surgery. We hypothesized that this temporary difference has little effect on the prognosis since the mean postoperative VAS scores of all patients were at low levels. Besides, no statistical differences in VAS scores were found before removing the internal fixation in all groups. In summary, the findings indicated that different internal fixation methods have similar radiographical and functional results.

In conclusion, ORIF with buttress plates and/or lag screws via a posterolateral approach can be an optimal approach in the treatment of posterior malleolus fractures. The technique provides excellent reduction and satisfactory clinical outcomes, with few postoperative complications.

\section{Declarations}

Funding Not applicable

\section{Competing interests}

The authors declare that they have no competing interests.

\section{Availability of data and material}

The anonymised data that support the findings of this study are available from the corresponding author upon reasonable request.

\section{Ethical approval}

Approval for the study was obtained from our Institutional Review Board

\section{Consent for publication}

Not applicable.

\section{Authors' contributions}


All authors have been involved with this study since inception. YL and LZY undertook study design, data analysis,wrote the final paper. YG undertook ethical applications. ZJG, LHF, ZXQ,XL and LJB all aided in study design, data collection and analysis, writing and proof reading. The manuscript has been read and approved by all named authors.

\section{Acknowledgements}

We would like to thank participants for giving their time and sharing their experiences.

\section{References}

1. Court-Brown CM, McBirnie J, Wilson. G(1998)Adult ankle fractures-an increasing problem? Acta orthopaedica Scandinavica. 69(1): 43-47. doi: 10.3109/17453679809002355.

2. McDaniel WJ, Wilson F. C(1977)Trimalleolar fractures of the ankle. An end result study. CLIN ORTHOP RELAT R. (122): 37-45.

3. Mingo-Robinet J, López-Durán L, Galeote JE. et al(2011)Ankle fractures with posterior malleolar fragment: management and results. The Journal of foot and ankle surgery: official publication of the American College of Foot and Ankle Surgeons. 50(2): 141-145. doi: 10.1053/j.jfas.2010.12.013.

4. Drijfhout Van Hooff CC, Verhage SM, Hoogendoorn JM. Influence of fragment size and postoperative joint congruency on long-term outcome of posterior malleolar fractures. FOOT ANKLE INT. 2015;36(6):673-8. doi. 10.1177/1071100715570895.

5. Berkes MB, Little MT, Lazaro LE. et al(2013)Articular congruity is associated with short-term clinical outcomes of operatively treated SER IV ankle fractures. The Journal of bone and joint surgery. American volume. 95(19): 1769-1775. doi: 10.2106/JBJS.L.00949.

6. Gardner MJ, Brodsky A, Briggs SM. et al(2006)Fixation of posterior malleolar fractures provides greater syndesmotic stability. CLIN ORTHOP RELAT R. 447: 165-171. doi:

10.1097/01.blo.0000203489.21206.a9.

7. Ketz J, Sanders. R(2012)Staged posterior tibial plating for the treatment of Orthopaedic Trauma Association 43C2 and 43C3 tibial pilon fractures. J ORTHOP TRAUMA. 26(6): 341-347. doi: 10.1097/BOT.0b013e318225881a.

8. Jehlicka D, Bartonícek J, Svatos F, et al(2002)[Fracture-dislocations of the ankle joint in adults. Part I: epidemiologic evaluation of patients during a 1-year period]. ACTA CHIR ORTHOP TR. 69(4): 243247.

9. Bartoníček J, Rammelt S, Tuček M. et al(2015)Posterior malleolar fractures of the ankle. European journal of trauma and emergency surgery: official publication of the European Trauma Society. 41(6): 587-600. https://doi: 10.1007/s00068-015-0560-6.

10. Verhage SM, Hoogendoorn JM, Krijnen P, et al(2018)When and how to operate the posterior malleolus fragment in trimalleolar fractures: a systematic literature review. ARCH ORTHOP TRAUM SU. 138(9): 1213-1222. https://doi: 10.1007/s00402-018-2949-2. 
11. van den Bekerom MP, Haverkamp D, Kloen. $P(2009)$ Biomechanical and clinical evaluation of posterior malleolar fractures. A systematic review of the literature. The Journal of trauma. 66(1): 279-284. doi: 10.1097/TA.0b013e318187eb16.

12. De Vries JS, Wijgman AJ, Sierevelt IN, et al(2005)Long-term results of ankle fractures with a posterior malleolar fragment. The Journal of foot and ankle surgery: official publication of the American College of Foot and Ankle Surgeons. 44(3): 211-217. doi: 10.1053/j.jfas.2005.02.002.

13. Macko VW, Matthews LS, Zwirkoski P, et al(1991)The joint-contact area of the ankle. The contribution of the posterior malleolus. The Journal of bone and joint surgery. American volume. 73(3): 347-351.

14. Haraguchi $\mathrm{N}$, Haruyama $\mathrm{H}$, Toga $\mathrm{H}$, et al(2006)Pathoanatomy of posterior malleolar fractures of the ankle. The Journal of bone and joint surgery. American volume. 88(5): 1085-1092. doi: 10.2106/JBJS.E.00856.

15. Bartoníček J, Rammelt S, Tuček. M(2017)Posterior Malleolar Fractures: Changing Concepts and Recent Developments. FOOT ANKLE CLIN. 22(1): 125-145. doi: 10.1016/j.fcl.2016.09.009.

16. O'Connor TJ, Mueller B, Ly TV, et al(2015)"A to p" screw versus posterolateral plate for posterior malleolus fixation in trimalleolar ankle fractures. J ORTHOP TRAUMA. 29(4): e151-e156. doi: 10.1097/BOT.0000000000000230.

17. Vidović D, Elabjer E, Muškardin I, et al(2017)Posterior fragment in ankle fractures: anteroposterior vs posteroanterior fixation. INJURY.: S65-S69. https://doi: 10.1016/S0020-1383(17)30743-X.

18. Tornetta P, Ricci W, Nork S, et al(2011)The posterolateral approach to the tibia for displaced posterior malleolar injuries. J ORTHOP TRAUMA. 25(2): 123-126. doi: 10.1097/BOT.0b013e3181e47d29.

19. Verhage SM, Boot F, Schipper IB. et al(2016)Open reduction and internal fixation of posterior malleolar fractures using the posterolateral approach. The bone \& joint journal. (6): 812-817. doi: 10.1302/0301-620X.98B6.36497.

20. Branca Vergano L, Monesi M, Vicenti G, et al(2020)Posterior approaches in malleolar fracture: when, why and how. J BIOL REG HOMEOS AG. 34: 89-95.

21. Rammelt S, Heim D, Hofbauer LC, et al(2011)[Problems and controversies in the treatment of ankle fractures]. Der Unfallchirurg. 114(10): 847-860. https://doi: 10.1007/s00113-011-1978-x.

22. Rammelt S, Zwipp H, Mittlmeier T(2013)[Operative treatment of pronation fracture-dislocations of the ankle]. OPER ORTHOP TRAUMATO 25(3): 273-91, 291-293. https://doi: 10.1007/s00064-0130235-6.

23. Erdem MN, Erken HY, Burc $\mathrm{H}$, et al(2014)Comparison of lag screw versus buttress plate fixation of posterior malleolar fractures. FOOT ANKLE INT. 35(10): 1022-1030. doi:

10.1177/1071100714540893.

24. Bennett C, Behn A, Daoud A, et al(2016)Buttress Plating Versus Anterior-to-Posterior Lag Screws for Fixation of the Posterior Malleolus: A Biomechanical Study. J ORTHOP TRAUMA. 30(12): 664-669. doi: 10.1097/BOT.0000000000000699.

25. Li M, Collier RC, Hill BW, et al(2017)Comparing Different Surgical Techniques for Addressing the Posterior Malleolus in Supination External Rotation Ankle Fractures and the Need for Syndesmotic 
Screw Fixation. The Journal of foot and ankle surgery: official publication of the American College of Foot and Ankle Surgeons. 56(4): 730-734. doi: 10.1053/j.jfas.2017.01.053.

26. Zhou Q, Lu H, Wang Z. et al(2017)Posterolateral Approach With Buttress Plates and Cannulated Screw Fixation for Large Posterior Malleolus Fractures. The Journal of foot and ankle surgery: official publication of the American College of Foot and Ankle Surgeons. 56(6): 1173-1179. doi: 10.1053/j.jfas.2017.05.028.

\section{Tables}

Due to technical limitations, tables are only available as a download in the Supplemental Files section.

\section{Figures}
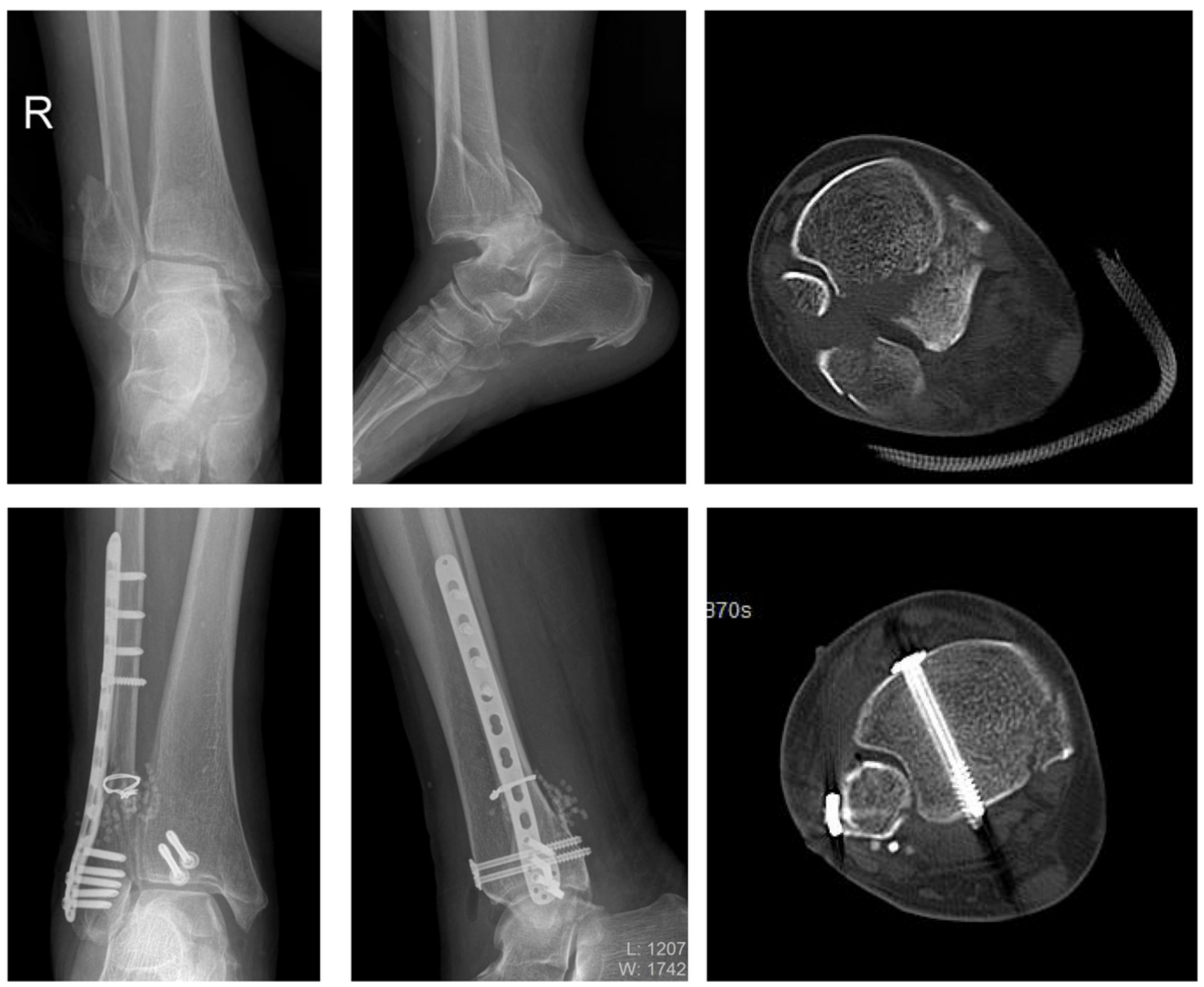

Figure 1 
AP, lateral ankle views and CT scans of a trimalleolar ankle fracture with a AP lag screw fixation of the posterior malleolus.
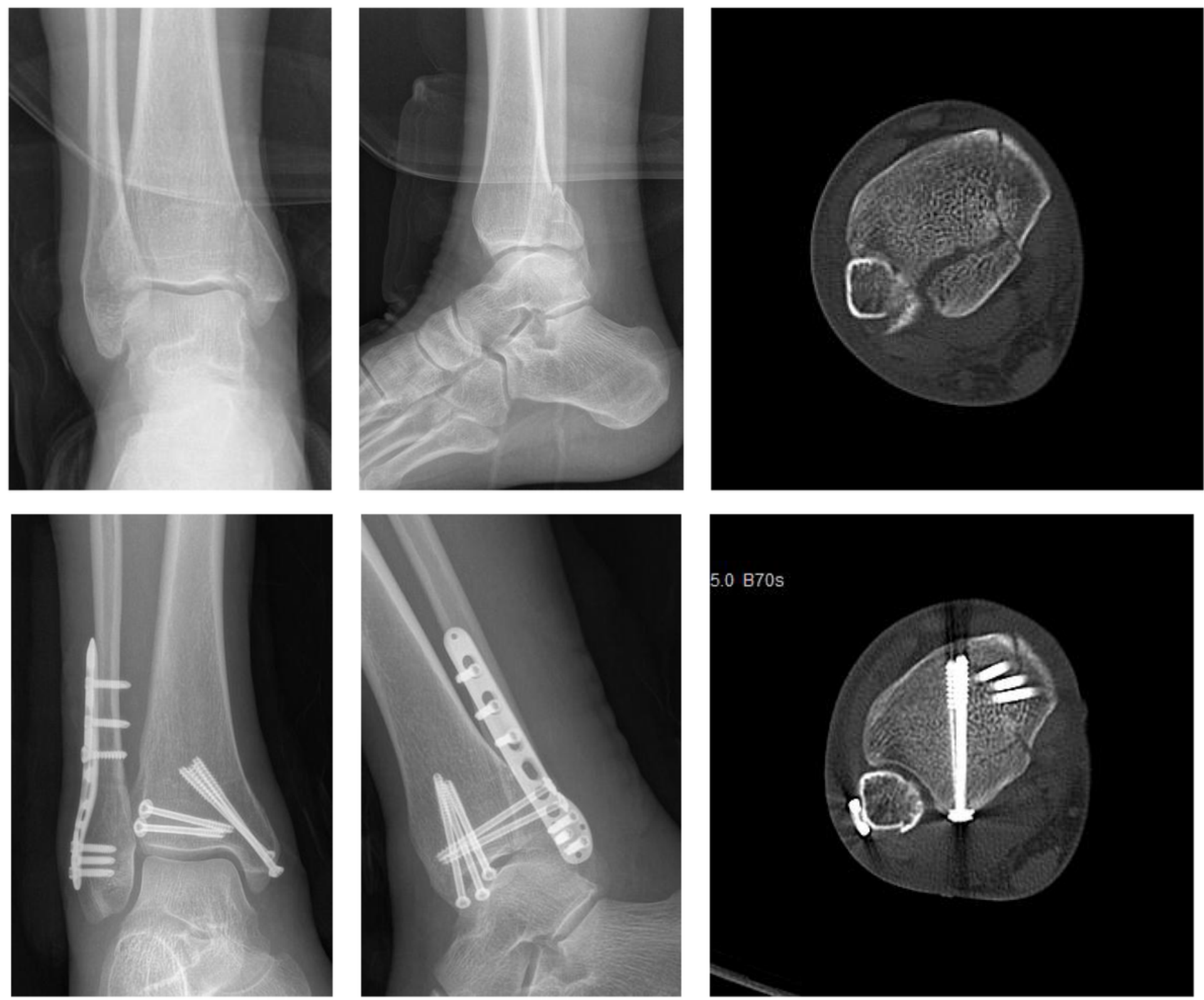

Figure 2

AP, lateral ankle views and CT scans of a trimalleolar ankle fracture with a PA lag screw fixation of the posterior malleolus. 

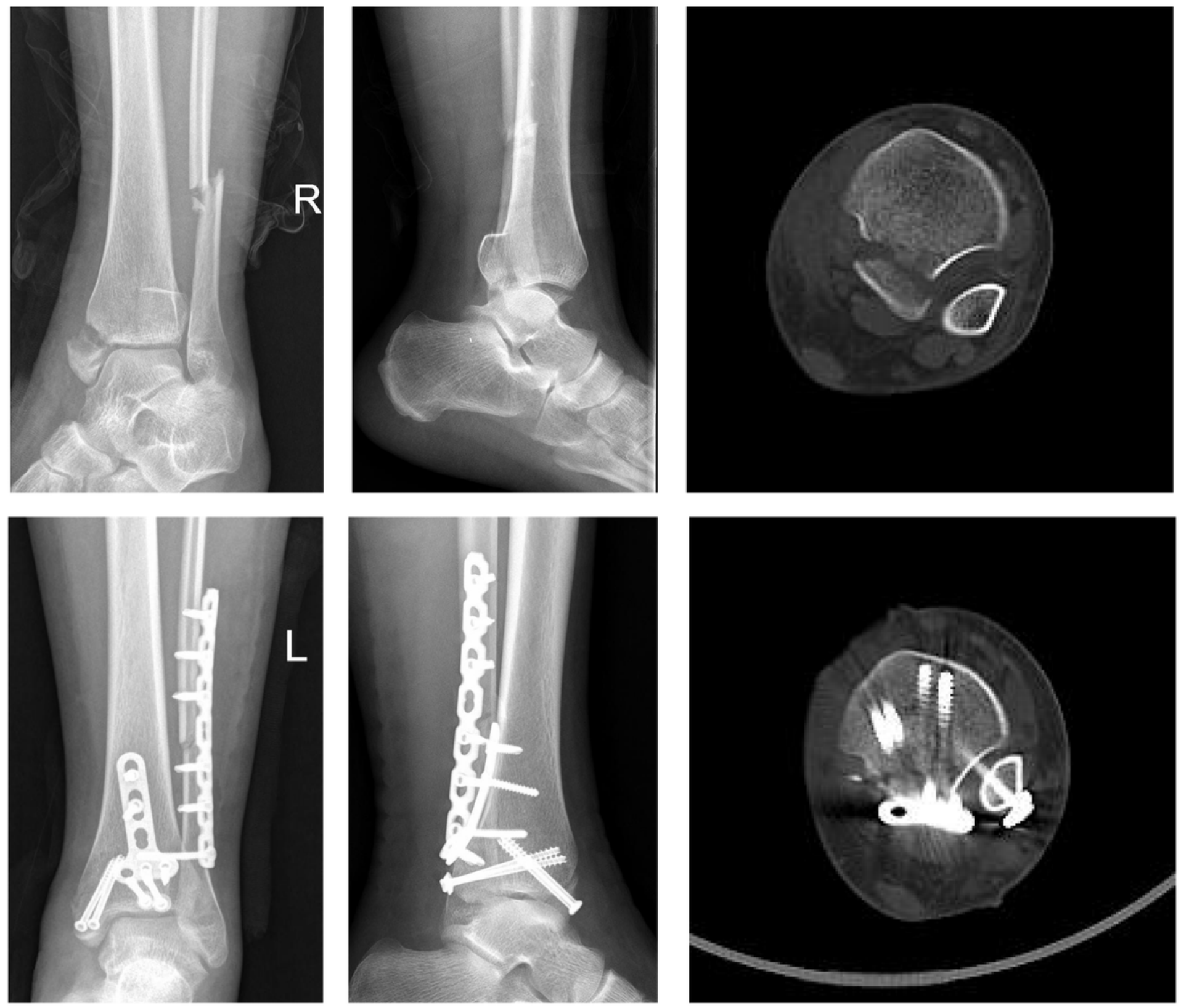

\section{Figure 3}

Radiographs of the posterior malleolus fixing with a buttress plate and two lag screws

\section{Supplementary Files}

This is a list of supplementary files associated with this preprint. Click to download.

- table14.pdf 